\title{
Image Segmentation Using an Improved Hybrid Modelling
}

\author{
Charlotte Ama Mensah \\ ICT Tutor \\ Bibiani Senior High Tech. Sch. \\ Ghana Education Service
}

\author{
C. Osei - Bonsu \\ Computer Science Department \\ Kwame Nkrumah University of \\ Science \&Tech \\ Kumasi, Ghana
}

\author{
Owusu Nyarko-Boateng \\ Innerjoy Digital Systems \\ Sunyani, Ghana
}

\begin{abstract}
The research was conducted with the main purpose of developing a proposed algorithm; Charlotte Ama Mensah Segmentation Algorithm (CAMSEG) that combines K-Means and PSO clustering algorithms under the supervision of the Otsu Algorithm, which acts as the intelligent part of the algorithm to find the threshold value of an image and with respect to the threshold value, the CAMSEG algorithm selects one of the two algorithms to start the optimization process and will complete the process with the other algorithm (that is, either KM/PSO or PSO/KM).The study makes use of the JAVA programming language to implement the following five algorithms; K-Means, PSO, hybrid K-Means PSO, hybrid PSO K-Means and CAMSEG. The CAMSEG algorithm is suggested because the K-Means algorithm works best with images whose threshold values are less than or equal to 180 and so based on this the CAMSEG algorithm chooses to begin the segmentation process with K-Means for all images with threshold value less or equal to 180.This is done by using the Otsu's algorithm to find the threshold value of the image, then based on the threshold value, the algorithm chooses which of the two algorithms to begin the segmentation with. After testing all algorithms with sample images, the general implication is that, it is possible to allow one hybrid algorithm to automatically decide which of the two algorithms K-Means or PSO to start the segmentation process with and end with the other algorithm. It has therefore been concluded that not all images can be conveniently segmented with either hybrid KM/PSO or PSO/KM to give effective results since images have different threshold values.
\end{abstract}

\section{Keywords: Clustering, Segmentation, K-Means, CAMSEG, Algorithm}

\section{INTRODUCTION}

Vision is indisputably the most important of all the perceptual mechanisms that humans possess. The subconscious way that humans often look, interpret and eventually act upon what is seen, however, belies the intricacy of the visual perception. When human beings use the eyes to look at an object, a system in their brains try to segment that complex object immediately, into a simple object that contains numerous areas. This is basically the process of further dividing an image into basic parts and extracting the parts of importance, which are the areas of the object. When technology was introduced, computer vision has progressively developed into a bigger part of the society. It has been used in several applications such as medical imaging, video surveillance, machine vision, traffic control systems, object detection, among many other useful applications. Hence, image segmentation is one of the basics of computer vision and it represents the first step in image analysis and pattern recognition.

\subsection{Problem Statement}

A digital image is a discrete two-dimensional function, $f(x, y)$, which has been quantized over its domain and range

(Gray and Neuhoff, 1998). All image processing operations generally aim at a better recognition of objects of interest, that is, at finding suitable local features that can be distinguished from the objects and from the background. The next step is to check each individual pixel to see whether it belongs to an object of interest or not. This operation is called segmentation and it produces a binary image. A pixel has the value of one, if it belongs to the object; otherwise it is zero (Singh and Singh, 2010). Segmentation involves partitioning an image into pixels which are homogeneous with respect to some criterion. Image segmentation is one of the most important and classical problems in image analysis. It should partition the image into disjoint regions, uniform according to some given features like grey level, color or texture. The segmentation process can rely on the uniformity of the features within the regions or on edge information (discontinuities in the feature space). In any case, the result should be a balance between the possibly noisy or incomplete input data and smoothness of the results best suited for further analysis (H. Nguyen et al, 2003).

Image segmentation is a classic problem in computer vision. It is a process that partitions the image pixels into meaningful groups so that we can achieve a compact representation of the image (Forsyth and Ponce, 2003).

\subsection{Objectives of the Study}

The main objective of the research is to develop the CAMSEG algorithm that combines the K-Means and PSO clustering algorithms under the supervision of the Otsu Algorithm. The Otsu Algorithm acting as the intelligent part of the algorithm finds the threshold value of an image and with respect to the threshold value; the CAMSEG algorithm chooses to begin the segmentation process with K-Means for all images with threshold value less or equal to 180 or PSO 
when it is otherwise and will complete the process with the other algorithm (that is, either KM/PSO or PSO/KM).

\subsubsection{Specific Objectives}

The specific objectives of the study are outlined below;

1. To establish the drawbacks of the K-Means algorithm and the PSO algorithm.

2. To examine how the K-Means and the PSO algorithms are separately implemented and applied to image segmentation.

3. To study how effective a hybrid algorithm of KMeans and PSO will be used enhance clustering image segmentation.

4. To study the incorporation of Otsu's Algorithm into a hybridized K-Means and PSO algorithm to make clustering segmentation become more effective and efficient.

\subsection{Significance of the Study}

The following areas of human endeavor will benefit more from this research;

Medical Imaging: Image segmentation has played a very important role in most researches in medical image analysis. Having concise segmentations will greatly benefit medical personnel as well as patients to provide essential information for 3 - dimensional visualization, surgical planning, virtual surgery simulation, early disease detection as well as locating tumors and other pathologies.

Object Detection: Image segmentation is useful for object detection such as pedestrian detection, face detection (used mostly in criminal identification and well as security systems) and also the location of objects in satellite images.

Security Purposes: Image segmentation is used in security systems for security purposes which may include fingerprint recognition, iris recognition as well as that of face recognition.

The research will also be beneficial to the traffic control system operators and also those in video surveillance systems.

\subsection{Main Approaches to Image Segmentation}

Image segmentation has been an important research area and numerous segmentation methods have been examined in this literature. Being a so well studied area, a number of approaches have been proposed. The classification of the different techniques into groups has been an inconsistent task. There have been several alternative classifications based on different authors. Indeed the classification and its underlying motivation depend on the author and sometimes the content.

1. The reports on Image Segmentation Studies conducted by the College of Engineering, Mathematical and Physical Sciences of the University of Exeter in 2001 identified that the techniques are divided into histogram, edge, region, probabilistic and clustering based methods.

2. According to Sonka, Hlavac and Boyle (1999), the segmentation techniques are also divided into histogram, edge and region based techniques.

3. In this documentation, the classification adopted was propounded by Ying Ho and Zheng Lee, 2003. The segmentation approaches were classified into four, which are;

- region-based approaches

- $\quad$ edge-based approaches
- $\quad$ split and merge approaches

- Clustering-based methods.

Brief description of each of these approaches is given in the subsequent sub-sections.

\subsubsection{Region - Based Methods}

In region-based methods, the input image is divided into several connected regions by grouping the adjacent pixels of close intensity levels (Ying Ho and Zheng Lee, 2003). The aim is the recognition of regions that satisfy some predefined homogeneity criteria. Neighboring regions are further merged due to their homogeneity or sharpness of region boundaries.

Region-based segmentation is a method for finding the region directly. The two main constraints to region-based segmentation are as follows;

$$
\bigcup_{i=1}^{n} R_{i}=R 1.1
$$

where

$$
R_{i} \text { is a connected region, } i=1,2, \ldots, n \text {. }
$$

This means that the union of all regions must be equal to the original image $\mathrm{R}$.

$$
R_{i} \cap R_{j}=\emptyset, \text { for } i, j=1,2, \ldots, n \quad \text { if } i \neq j
$$

The second constraint also means that each and every pixel must belong to only one particular region (Ying Ho and Zheng Lee, 2003).

\subsubsection{Edge - Based Methods}

Edge-based methods of image segmentation are standing on edge detection which is a well-expanded field in image processing. Since there is often a sharp alteration at intensity of the object boundaries, these boundaries can be considered as the edges. However, closed region boundaries must be determined to detect image objects and the detected edges are the boundaries between objects (Gonzales and Woode, 1981). Therefore, edge detection techniques have been used as the basic step of other segmentation methods. In this approach, image edges are recognized and then linked into lines that indicate the borders of image objects (Gonzales and Woode, 1981).

Candidate edges are extracted by thresholding the gradient or Laplacian magnitude (Canny, 1986). It is also possible that the edges that are identifies by edge detection algorithms are sometimes not continuous. In solving this, a number of evolutionary algorithms are proposed to detect well-localized and continuous thin edge based on optimization of edge configurations (Gudmundsson et al, 1998).

\subsubsection{Split and Merge Approaches}

As the name suggests, these are segmentation methods that have two procedures. The image is segmented into several regions and inappropriate regions are merged into the correct 
ones. In split and merge methods, an input image is segmented into set of homogeneous regions in advance (Ying Ho and Zheng Lee, 2003). Then similar neighbouring regions are merged according to certain homogeneity features. In the merge phase, each inappropriate region needs to be merged with one of their neighbours. As a result, Region Adjacency Graph (RAG) is used to determine neighbouring regions (Bhandarkar and Zhang, 1999). RAG is updated to represent the new regions and their correct neighbour after each merge procedure's iteration.

\subsubsection{Clustering Methods}

Clustering is defined as the process of putting objects into groups based on the similarity in their members. According to Ergin, 2001;

The following definitions may be functional as far as image segmentation is concerned:

i. A cluster is a set of entities, which are alike, while entities from different clusters are not alike.

ii. A cluster is an aggregation of points in the test space such that the distance between any two points in the cluster is less than the distance between any point in the cluster and any point not in it.

iii. Clusters may be described as connected regions of a multi-dimensional space containing a relatively high density of points, separated from other such regions by a region containing relatively low density of points.

Research into clustering algorithms has been beneficial in many applications, especially in the area of data mining and pattern recognition. Clustering methods are divided into two; hierarchical and partitional (Bhalerao. 1991). Within each of these two categories are a number of different algorithms for finding the clusters.

\subsubsection{Hierarchical Clustering}

Hierarchical clustering techniques are based on the use of a proximity matrix indicating the similarity between every pair of data points to be clustered. The end result is a tree of clusters, called a dendrogram representing the nested grouping of patterns and similarity levels at which grouping change. It proceeds successively by either merging clusters into larger ones (agglomerative, bottom-up), or by splitting larger clusters (divisive, top-down). By cutting the dendrogram at a desired level, a clustering of data items into disjoint group is obtained. The clustering methods differ in regards to the rules by which two small clusters are merged or a large cluster is split. Some of the hierarchical algorithms include Cure, Chameleon and Cobweb (Bhalerao, 1991).

A hierarchical clustering which models the blurring effect of lateral retinal interconnections to stimulate the human visual system based on scale space theory was proposed by Leung et al, 2000. The following statement gives an idea about this as described by Leung et al (2000);

"In this approach, a dataset is considered as an image with each light point located at a datum position. As this image was blurred, smaller light blobs merges into bigger ones until the entire image became one light blob at a low level resolution. The blurring process produces a family of clustering along the hierarchy by identifying each blob with a cluster" (Leung et al, 2000)

\subsubsection{Partitional Clustering}

Partitional clustering algorithms break up the image into a specific number of clusters. Partitional clustering algorithms are usually iterative algorithms that converge to local optima (Hamerly et al, 2002). A partition clustering is considered as a single partition while a hierarchical clustering is a nested sequence of partitions (Ergin, 2001).

Partitional clustering algorithms such as, K-Means has an advantage over hierarchical clustering algorithms, where there is a partition of the data points which optimizes some criterion functions. In the hierarchical clustering, if a data point is assigned to a particular cluster, it cannot be changed. As a result, if a data point is not correctly assigned to a particular cluster at an early stage, there will be no way to correct the error. But the partitional clustering techniques also have a problem with regards to how to determine the number of cluster, k (Bhalerao, 1991).

\subsection{K - Means Clustering Algorithm}

McQueen first introduced the K-means algorithm in 1967 as an unsupervised clustering algorithm that partitions a data set into a specific number of clusters. The K-Means algorithm is based on minimizing the performance index which is defined as the sum of the squared distances from all points in a cluster domain to a cluster center (Tou and Gonzalez, 1974).

The K-Means algorithm is one of the partitioning based, nonhierarchical clustering methods. Given a set of numeric objects $\mathrm{X}$ and an integer number $\mathrm{k}$, the K-Means algorithm searches for a partition of $\mathrm{X}$ into $\mathrm{k}$ clusters that minimizes the within groups sum of squared errors. The K-Means algorithm starts by initializing the $\mathrm{k}$ cluster centers. The input data points are then allocated to one of the existing clusters according to the square of the Euclidean distance from the clusters, choosing the closest. The mean (centroid) of each cluster is then computed so as to update the cluster center. This update occurs as a result of the change in the membership of each cluster. The processes of re-assigning the input vectors and the update of the cluster centers is repeated until there are no more change in the value of any of the cluster centers. .

\subsection{PSO - Fuzzy System Approaches in Image Segmentation In 2008, Masooleh et al. suggested a fuzzy system in combination with PSO for image segmentation. A sugeno fizzy system was used in this method. Each pixel was allocated a colour class by applying a set of fuzzy rules. Large number of fuzzy rules is the main problem of fuzzy system. For this reason, PSO was used and it automatically produced the smallest number of optimum fuzzy rules and generated the optimized membership function.}


A color image segmentation using a complete learning PSO based fuzzy system was presented in 2009 by Puranik et al. In this improved version of PSO, all particles' Pbest were used to update the velocity of other particles. A fitness function which rates the optimality of each particle was also used. This new PSO was an optimization process which found the optimal fuzzy rules and the membership function. A set of fuzzy rules was assigned to each particle. Each particle was expected to try to maximize the fitness function during the process. This approach discouraged the premature convergence of original PSO. In addition, the HSL colour space was used in the proposed approach because it can be presented in three-dimensional for fuzzy colour classification.

Gopal et al. (2010) presented two phase of MRI segmentation. The first phase includes pre-processing and enhancement. To remove labels and $\mathrm{x}$-ray marks from MRI images, a tracking algorithm was proposed. Along with this, a median filter was used to remove high frequency components from MRI images. The fuzzy c-means (FCM) was used to calculate the adaptive threshold, after PSO which automatically determines the optimal threshold value of the given image to select initial cluster seed point.

A muli-elasic exponential PSO which was hybridized with fuzzy system to perform segmentation of colored images was illustrated by Murusegan et al. (2001). Multi elastic exponential PSO is a blend of multi elastic PSO and exponential PSO. This approach made use of a kernel induced similarity measure for searching global best of PSO. The standard PSO converges very early in search space but the new approach helps to avoid this convergence characteristic of PSO. Alternatively, exponential PSO prevents the particles from stagnation of local optima by changing the inertia weight exponentially. This hybridized PSO was used to locate the optima fuzzy rules and membership function. Each particle sought to maximize the fitness function and the best fuzzy rule was selected for image segmentation.

\subsection{PSO - Genetic Algorithm Approaches in Image Segmentation}

Due to the sensitivity to noise by the traditional Fuzzy CMeans (FCM) clustering algorithm, one of the basic methods is low pass filtering of an image and then applying the FCM clustering algorithm. The problem with this approach was that it could lead to loss of the important details present in an image. To overcome this problem, an essential FCM clustering algorithm was proposed by Shen et al. (2005). The parameter optimization is an important parameter that could affect the performance of FCM clustering method. Forouzanfer et al. (2010), proposed a breeding swarm algorithm to help discover optimum attraction parameters. This algorithm combined the strengths of both genetic and PSO algorithm. The algorithm was designed so that PSO supports local search and genetic algorithm performs global search. The experimental results indicated that this proposed breeding swarm with FCM cluttering algorithm was an excellent approach for segmenting MRI images.
An approach to image segmentation using hybrid technique based on PSO and genetic algorithm was described by Kole et al. (2010). The optimal number of clusters was determined by using the PSO based dynamic clustering. The genetic algorithm further used this information to improve the final result of the PSO based method. The best result was obtained finally by comparing their individual validity indices with that of (Turi, 2001) and the data is partitioned accordingly.

2.4.1 PSO - Wavelet Approaches in Image Segmentation Particles are normally prematurely attracted to the local attractor in a standard PSO. An inertia adaptive PSO and wavelet mutation algorithm which assisted the particles to break away from local minima and resulted in an increase in the speed of the segmentation process was proposed by Wei et al. (2010). Fuzzy entropy was used to calculate the fitness function of the particles in the swarm. Two dynamical regimes are used to govern the motion of the particles. The inertia adaptive PSO was used to sample particles if there was an improvement in the fitness function of the particles from iteration to iteration. The wavelet mutation had been proposed if there is no improvement, because that because that will result in stagnation. Exhibiting a fine tuning ability is one of the advantages of wavelet mutation.

An illustration on how PSO could be successfully integrated with wavelet mutation and provide a more effective approach to resolve the stagnation problems was given by De et al. (2011). The entropy maximization was employed to get the expert knowledge of the probable threshold grey level range for stagnation of MRI images after their normalized histograms had been initially obtained. The hybrid PSO and wavlet mutation is used to optimize the initial value of the threshold. The region of interest was obtained using this threshold value. Finally the segmented MRI images with lesions were obtained by employing a variable mask on region of interest.

2.4.2 PSO-Clustering Approaches in Image Segmentation A method that uses FCM cluttering together with PSO was proposed by Chun et al. (2008). The main purpose of FCM clustering is to locate cluster centers that maximizes the similarity function and minimizes the dissimilarity function. The pixels are assigned to clusters using the PSO. The number and initial location of the center of the predetermined clustering affect the basic FCM algorithm.

Jing et al. (2010), proposed a fast FCM method with PSO for image segmentation. The number of clusters and the centre of the clusters was automatically determined by the PSO algorithm as an optimization process.

There is a difficulty in segmenting sonar images because they have low signal to noise ration. Liu et al. (2010) presented a PSO based fuzzy cluster algorithm for segmenting sonar images. This hybridization resulted in producing strong searching and high speed convergence ability. Additionally, the fuzzy measure and fuzzy integral were also calculated to compute the fitness. 
Jing et al. (2010) also presented an approach to fit clusters which were close to one another, this was necessary because the possibilistic c-means (PCM) algorithm is very sensitive to initialization and parameters. The complex computation and initial parameter sensitivity problems were solved by using the $\mathrm{t}$ - Particle Swarm Optimization ( $\mathrm{t}$ - PSO). This algorithm was targeted at getting an accurate segmentation. It was shown that the proposed algorithm was less influenced by the noise points and produced better results.

An illustration of how PCM could be integrated with PSO was presented by Zhang et al. (2011), they also provided a significant improvement on the efficiency of the segmentation. When compared to FCM, the PCM was more accurate because it overcame the relative membership problem of FCM in image segmentation. The PCM algorithm uses the mahalonolis distance, since it enhances the performance of the clustering algorithm.

Researchers observed that it was difficult to segment underwater images because they have low signal to noise ratio. It was also observed that the traditional FCM algorithm did not produce good results and it was very time consuming. Researchers Wang et al. (2011) therefore presented a segmentation algorithm based on histogram weighted FCM to solve this problem. The statistical behavior of histogram of grey images were considered, this presented a fast and effective FCM algorithm for underwater image segmentation.

\subsubsection{PSO - Rough Set Approaches in Image Segmentation} Feng et al. (2009) proposed an approach that uses rough set entropy with PSO. The authors segmented a grey-scale image by applying rough set entropy. The optimal threshold was obtained by using PSO and rough set entropy which was based on boundary conditions. Experimental results indicated that the proposed apgorithm was time efficient, the system became more stable and finally the sensibility of the algorithm to partition size image sub-piece was also low.

A hybrid rough set PSO for partitioning an image into different meaningful segments was presented by Behera et al. (2011). Each cluster was treated an interval or rough set in rough c-means algorithm. K-Means clustering algorithm was used for image pixels classification and that calculated the initial means and their positions in the clusters. The upper and lower bounds of the clusters were calculated after the cluster centers had been obtained. The cluster centers were upgraded using the rough set, and the parameters of rough c-means were tuned using PSO. In this approach, a statistical mathematical function by name Davies Bouldin (1979) index was used for the purpose of the fitness function in PSO. The result of the performance evaluation of PSO showed that the method reduced noisy spots and was less sensitive to noise.

\subsubsection{PSO - Neural Networks Approaches in Image} Segmentation

White blood cell image segmentation was presented by Yi et al. (2005), the algorithm incorporated an online training neural network. To begin with, a mean shift algorithm was employed to search the cluster centre. After that, the size of the training set was reduced by performing uniform sampling. Through the use of uniform sampling, the statistical data revealed that subset could represent the entire data set approximately. In addition, the PSO algorithm employed helped in faster convergence and the escape from local optimum.

Alamelumangai et al (2010) proposed a neutron - fuzzy filter for image enhancement, this was due to the fact that image enhancement and pre-processing are requisites for ultrasound images because they have low contrast and speckle noise. After pre-processing, an artificial neutron - fuzzy network and Eliminating PSO were used for image segmentation. The proposed algorithm was mainly a five layer network and the inputs were fuzzy values. The Eliminating PSO eliminated the weakest particle and searched for optimal solution. The algorithm helped in reducing the computational time without affecting the accuracy of the solution.

\subsection{K-MEANS}

The K-Means algorithm is started with an initial partition and assignment of patterns to clusters to reduce the error between the intensities of the pixels of a cluster and it's mean. The error tends to decrease as the number of iterations increases, as expected is minimized for a fixed number of clusters, $\mathrm{K}$ (Ergin, 2001).

\section{Sample Images}

In all, six (6) different images were used to test the algorithm. Figures 3.1a and 3.2a are sample Image1 and Image2 before segmenting with K-Means; the corresponding results after KMeans segmentation are shown respectively in Figures $3.1 \mathrm{~b}$ and $3.2 b$

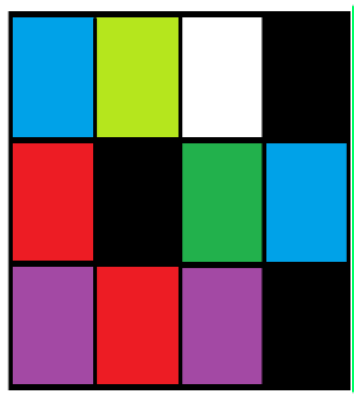

Before

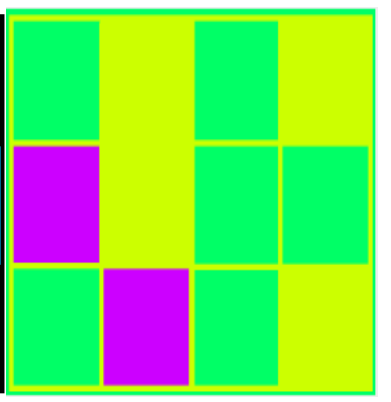

After
Figure 3.1a Sample Image1 Figure 3.1b Resultant Image 1

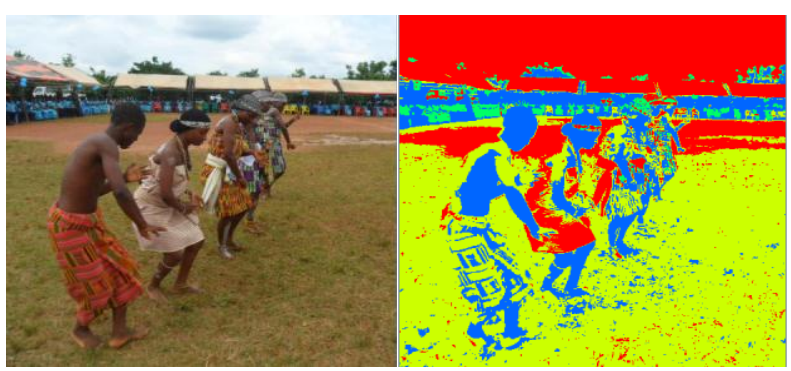


Before

After

Figure 3.2a Sample Image2 Figure 3.2b Resultant Image2

The simulation was further carried out with four (4) other algorithms. These include:
i. The PSO algorithm
ii. Hybrid PSO and K-Means algorithm
iii. K-means and PSO Hybrid algorithm
iv. The CAMSEG algorithm

\subsection{RESULTS AND DISCUSSIONS}

The results of two out of the six sampled images are used for segmenting all five models of the program, that is, K-Means, PSO, KMPSO, PSOKM and the CAMSEG algorithm. Two images, that is Image 1 and Image 2 were used for the test. The images and their results are presented in their matrix representations; histogram forms and finally the time durations used by all the algorithms will be discussed and compared. All these are done to establish the usefulness or otherwise of the CAMSEG algorithm.

\subsubsection{Discussion of Matrices}

\subsubsection{Summary of Image1 Matrices}

The table below gives a summary of the highest, lowest and modal pixels of image 1 being segmented with all five algorithms.

Table 4.1 Summary of Frequency Distribution of Imagel for all Five Algorithms

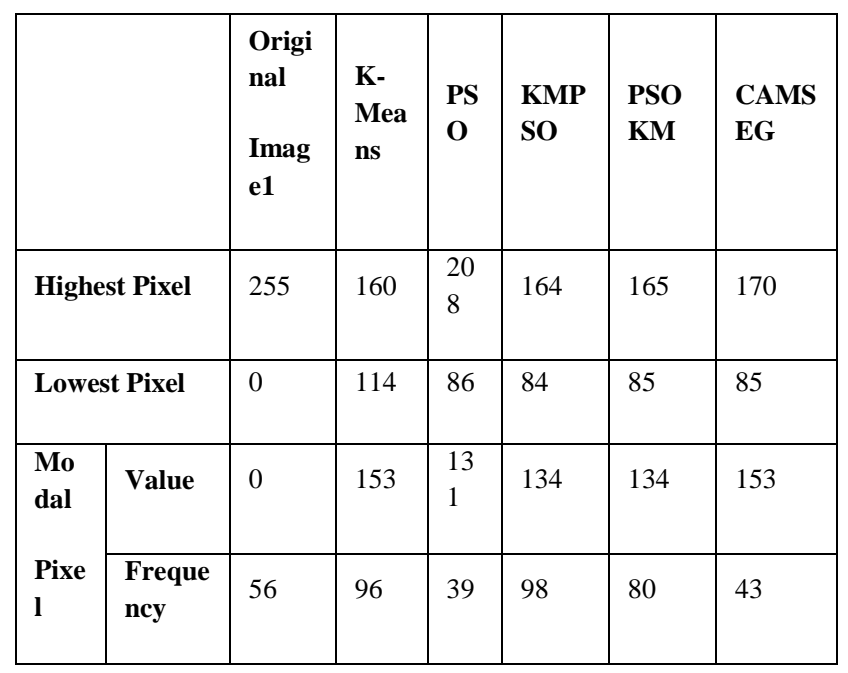

\section{Image1}

In examining the matrix produced by the original Image 1, it was observed that the values are small; ranging from a highest value of 255 to a lowest value of 0 . Table 4.5 shows that the highest frequency is 56 representing pixel value 0 . These values show that the original Image1 has a lot on the dark side than the bright side.

\subsection{K-Means and PSO Results}

The pixel values for the matrix produced by the K-Means segmentation ranges from 160 to 114 , which is rather on the high side. From Table 4.5 , it is shown that the pixel value with the highest frequency is 153 . This shows that this result of the K-Means is more of a mid-tone image. That is, this result of the K-Means is not as dark as the original image.

The PSO algorithm also produced an image whose matrix ranges from a highest pixel value of 208 to a lowest of 86 . Table 4.5 shows that the pixel with the highest frequency is 131 with a frequency value of 39 . This also shows a much brighter image compared to the original image but not brighter than the result of the K-Means algorithm.

\subsection{CAMSEG Algorithm}

The CAMSEG algorithm also produces a resultant image of Image 1 whose pixel values are relatively low but not lower than that of the original image, its highest pixel value is 170 and lowest pixel value being 85 . Table 4.1 shows that the pixel with the highest frequency is 153 with a frequency value of 43. This result is relatively bright; this can also be classified as a mid-tone range image though slightly brighter than the images produced by both KM-PSO and PSO-KM algorithms.

From these analyses, it can be said that the CAMSEG algorithm produced a relatively brighter result of Image 1compared to the results of the other two hybrid algorithms (PSO-KM and KM-PSO). Therefore it will be more prudent to choose the CAMSEG algorithm to segment this image, for instance in an object detection section, the CAMSEG algorithm will be more practical to use.

\subsection{Summary of Image2 Matrices}

Table 4.2 below gives a summary of the highest, lowest and modal pixels of image 2 being segmented with all five algorithms.

Table 4.2 Summary of Frequency Distribution of Image2 for all Five Algorithms

\begin{tabular}{|l|l|l|l|l|l|l|}
\hline & $\begin{array}{l}\text { Origi } \\
\text { nal }\end{array}$ & $\begin{array}{l}\text { K- } \\
\text { Mea } \\
\text { Imag } \\
\text { e2 }\end{array}$ & $\begin{array}{l}\text { PS } \\
\text { O }\end{array}$ & $\begin{array}{l}\text { KMP } \\
\text { SO }\end{array}$ & $\begin{array}{l}\text { PSO } \\
\text { KM }\end{array}$ & $\begin{array}{l}\text { CAMS } \\
\text { EG }\end{array}$ \\
\hline Highest Pixel & 253 & 161 & $\begin{array}{l}16 \\
7\end{array}$ & 163 & 155 & 170 \\
\hline
\end{tabular}




\begin{tabular}{|c|c|c|c|c|c|c|c|}
\hline \multicolumn{2}{|c|}{ Lowest Pixel } & 29 & 84 & 85 & 110 & 86 & 85 \\
\hline Mo & Value & 92 & 85 & $\begin{array}{l}13 \\
5\end{array}$ & 124 & 122 & 112 \\
\hline $\begin{array}{l}\text { Pixe } \\
1\end{array}$ & $\begin{array}{l}\text { Freque } \\
\text { ncy }\end{array}$ & 20 & 72 & 65 & 36 & 48 & 31 \\
\hline
\end{tabular}

\section{Image2}

The original Image2 has a highest pixel value of 253 and a lowest pixel value of 29 , from Table 4.6 , the pixel value with the highest frequency is 92 , with a frequency of 20 . Based on the pixel values represented in the matrix, it can be said that this image is fairly dark.

\subsection{K-Means and PSO}

The K-Means algorithm presented a resultant image whose highest pixel value is 161 and lowest pixel value is 84 , Table 4.2 shows that the pixel value with highest frequency is 85 , with a frequency value of 72 . Per the pixel values, this image is not so bright and not so dark too, the histogram language will say that it is in a mid-tone range. The PSO algorithm gave an image whose matrix has a highest pixel value of 167 and lowest being 85 . The pixel value with the highest frequency is 135 with a frequency value of 65 , as shown in Table 4.2. This image can also be said to be in the mid-tone range though slightly brighter than that of the K-Means. In comparing these two results, it is observed that K-Means produced an image that is slightly darker than the image produced by the PSO. Also, the matrices show that the PSO has its image distributed in a wider number of pixels compared with the K-Means.

\subsection{CAMSEG Algorithm}

The CAMSEG algorithm has a resultant image of Image2 having a matrix with the highest pixel value of 170 and lowest value of 85 . These pixel values are closely related to the PSO - K-Means algorithm than they are related to the K-Means PSO algorithm. This matrix has the pixel value of 112 having the highest frequency of 31 , as shown in Table 4.2; this also is closer to that of the PSO - K-Means matrix.

It is observed that the CAMSEG algorithm produced an image that has much brighter pixels compared to the original image. When compared to the two other hybrid algorithms (KMPSO and PSOKM), it is noted that the CAMSEG algorithm has darker pixels and also the modal pixel which is 112 has a relatively lower frequency compared to the two hybrid algorithms. In this instance as well, when it comes to making a choice out of these algorithms for segmentation, it will worthy to choose the CAMSEG algorithm since it produced a slightly better result compared to the other two hybrid algorithms.

\subsection{Time / Duration of the Image Segmentation}

The time/duration used by each algorithm to complete the segmentation of the two images (Image1 and Image2) is shown in Table 4.3 and Table 4.4 as they were run on two different computers with different specifications. Below are the specifications of the two computers.

Computer1: 32-bit registers, a RAM size of 2.00 gigabytes and a clock speed of $1.8 \mathrm{GHz}$.

Computer2:32-bit registers, a RAM size of 2.00 gigabytes and a clock speed of $3.20 \mathrm{GHz}$.

\subsubsection{Images and Duration of Segmentation Computer1}

The table and the chart below show the duration used by Computer 1 to segment Image 1 and Image 2 for all the five algorithms.

Table 4.3 Time Durations used by Computerl for the five Algorithms

\begin{tabular}{lcc}
\hline ALGORITHM & IMAGE1 & IMAGE2 \\
K-Means & $13.08 \mathrm{~m}$ & $13.06 \mathrm{~m}$ \\
PSO & $16.76 \mathrm{~m}$ & $1.772 \mathrm{~m}$ \\
K-Means - PSO & $16.27 \mathrm{~m}$ & $18.61 \mathrm{~m}$ \\
PSO - K-Means & $32.76 \mathrm{~m}$ & $12.90 \mathrm{~m}$ \\
CAMSEG Algorithm & $15.94 \mathrm{~s}$ & $1.363 \mathrm{~m}$ \\
\hline
\end{tabular}

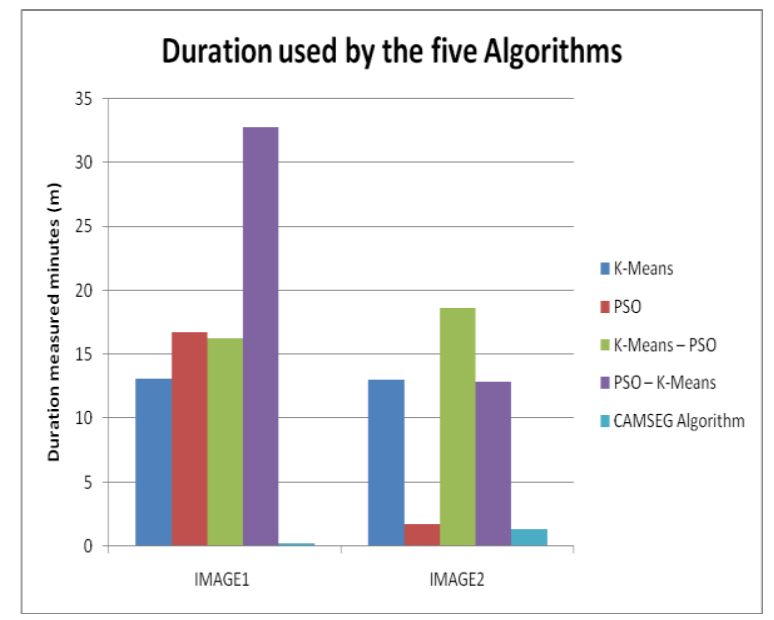

Figure 4.1 Duration used by Computer1 to run the five algorithms for segmenting Image 1 and Image 2. 
Table 4.4 Time Durations used by Computer2 for the five Algorithms

\begin{tabular}{lll}
\hline ALGORITHMS & IMAGE1 & IMAGE2 \\
K-Means & $1.323 \mathrm{~m}$ & $25.01 \mathrm{~s}$ \\
PSO & $1.434 \mathrm{~m}$ & $28.03 \mathrm{~s}$ \\
KPSO & $10.56 \mathrm{~m}$ & $4.629 \mathrm{~m}$ \\
PSOK & $7.273 \mathrm{~m}$ & $8.727 \mathrm{~m}$ \\
CAMSEG & $1.233 \mathrm{~m}$ & $20.05 \mathrm{~s}$ \\
\hline
\end{tabular}

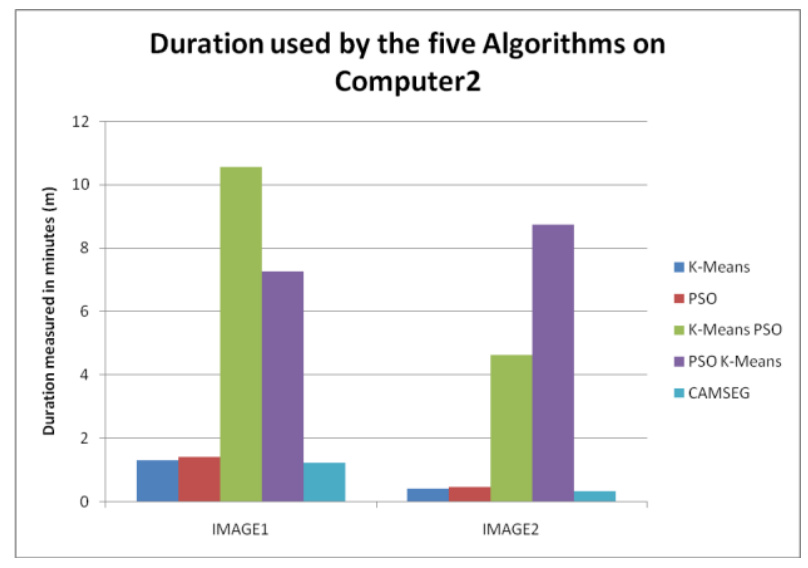

Figure 4.2 Duration used by Computer2 to run the five algorithms for segmenting Image1 and Image2

\subsection{Histogram of Images}

In this study, the resized images of 25 by 25 pixels are used for the histogram representation.

\section{Image1}

Figures 4.3 show Image 1 and its resultant images and the corresponding histograms.

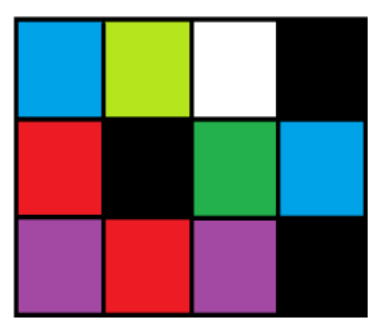

Original Image1

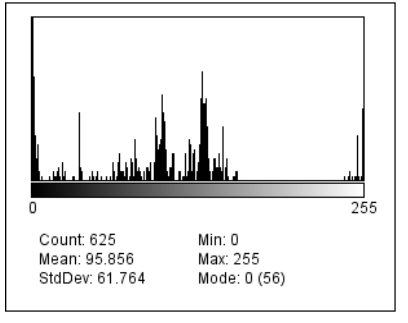

Histogram Representations
Figure 4.3 Original Image 1 and resultant K-means histograms

\subsection{Discussion of Duration used by the Algorithms}

From Table 4.3; it is observed that the algorithm with the longest amount of time for segmenting Image1 is the PSO-KMeans algorithm. Image2 also displays 18.61 minutes being the longest time used for segmentation and that is the KMeans - PSO algorithm.

It is also observed that in both Image1 and Image2; the CAMSEG algorithm used the least duration in the segmentation process, that is, 15.95 seconds and 1.363 minutes respectively. The same result is shown in Table 4.4 for computer2.It can therefore be concluded that the CAMSEG algorithm is more efficient in relation to time or duration needed for segmentation processes, that is, the CAMSEG algorithm is best when segmentation efficiency is being considered.

However, since efficiency does not automatically indicate effectiveness, it cannot be conclusively indicated that the CAMSEG algorithm is more effective than the other algorithms by merely considering just the duration it uses to segment the images without considering other areas to determine effectiveness of the CAMSEG algorithm. It is for this reason that the section that follows examines the histograms of the images.

\subsection{Summary}

The main objective of the research was to develop the CAMSEG algorithm that combines the K-Means and PSO clustering algorithms under the supervision of the Otsu Algorithm, with the aim of using the Otsu Algorithm to act as the intelligent part of the algorithm to find the threshold value of an image and with respect to the threshold value the CAMSEG algorithm selects one of the two algorithms to start the optimization process and will complete the process with the other algorithm (that is, either KM/PSO or PSO/KM).

The study examined the K-Means algorithm alone, the PSO algorithm alone, the hybridization of the two algorithms; the hybrid of the K-Means followed by the PSO algorithm and then the hybrid of the PSO algorithm followed by the KMeans algorithm.

The study further examined how to hybridize the two aforementioned algorithms where there will be an atomization, that is, the algorithm should automatically decide which of the two algorithms to start the segmentation process with and which one to be followed. By this, there was a need to find the threshold value of the image for this automatic decider, therefore the Otsu's algorithm for finding the threshold value was adopted to find the threshold value of the 
input image. All the algorithms were then implemented with the JAVA programming language and six sample images were used to segment all five algorithms. The resultant images of two out of the six were analyzed by examining their matrices; histograms and the duration taken to segment all images were noted.

\subsection{Conclusion}

From the histograms discussions, it can be concluded that the CAMSEG algorithm, in both images, produced much better images especially when compared to the two hybrid algorithms (K-Means PSO and PSO K-Means ) because its standard deviation values are much larger in both images indicating pixel values that are widely spread out. Also, the CAMSEG used the least duration in segmenting the two images as discussed above. It can therefore be concluded that the CAMSEG algorithm is more efficient in relation to time and quality of image in terms of histogram analysis.

The general conclusion is that, it is possible to use a single algorithm (CAMSEG Algorithm) to automatically decide which of the two algorithms K-Means or PSO to start the segmentation process and end with the other algorithm.

It can be concluded that, the CAMSEG algorithm has given better results showing the effectiveness and efficiency of the algorithm on both Image1 and Image 2 in all the different ways through which the results have been analyzed.

\subsection{Recommendation}

For further study, it is recommended that one may consider a more complex algorithm that will incorporate correcting the resultant image based on what it will be used for, especially in the field of computer visions. This research can also be further directed towards incorporating other image segmentation methodology like a patitional algorithm to make the segmentation more enhanced, especially if it is a research to segment images in the medical field. Also, the work can consider using more complex images such as 3-dimensional images.

\section{REFERENCES}

1) Alamelumangai, N. and Devishree, J.(2010). PSO Aided Fuzzy Inference System for Ultrasound Image Segmentation. International Journal of Computer Applications, vol. 7, No. 14, pages 16-20.

2) Behera, H. S., (2011). Segmentation and Classification using Heuristic HRSPSO. International Journal of Soft Computing and Engineering (IJSCE), Vol. 1, Issue 3, pages 66-69

3) Bhalerao,H. A. (1991). Multiresolution Image Segmentation, PhD Thesis, University of Warwick.- 21. Image Processing (ICALIP), pages 13651368.

4) De, A., Bhattacharjee, A. K., Chanda, C. K. and Maji, B. (2011). MRI Segmentation using Entropy Maximization and Hybrid PSO with Wavelet Mutation. World
Congress on Information and Communication Technologies (WICT), pages 362-367.

Science and Applications (ICISA), ( pages 1-7).

5) Ergin, M. S. (2001). "Scene Segmentation Comparative Analysis", MS Thesis, METU

6) Forouzanfar, M,. Forghani, N. and Teshnehlab, M. (2010). Parameter Optimization of Improved Fuzzy C-Means Clustering Algorithm for Brain MR image segmentation. Engineering Applications of Artificial Intelligence, Elsevier, vol. 23, Issue 2, pages 160-168.

7) Forsyth, D. and Ponce, J. (2003). Computer Vision A Modern Approach. Prentice Hall.

8) Gonzalez, R. C. and Woode, R. E. (1981). Realtime Digital Image Enhancement. Digital Image Processing vol. 69, No. 5.

9) Gopal, N.N. and Karnan, M. (2010).Diagnose Brain Tumor through MRI using Image Processing Clustering Algorithms such as Fuzzy C Means along with Intelligent Optimization TechniquesInternational Conference and Computing Researcsh (ICCIC), pages 1-4.

10) Gudmundsson, M., El-Kwae, E. A. and Kabuka, M. R. (1998). Edge Detection in Medical Images using A Genetic Algorithm. Medical Images, Vol 17, No. 3, pages 469 474.

11) Hamerly, G. and Elkan, C. (2002). Alternatives to the K-Means Algorithm that find

Better Clustering . In Proceeding of the ACM Conference on Information and Knowledge Management (CIKM - 2002), ( pages 600 607).

12) Hartigan, J. A. (1975). Clustering Algorithms. New York: John Wiley and Sons, Inc.

13) Jing, Z. and Bo, L. (2010). Image Segmentation using Fast Fuzzy C Means based on

PSO. 3rd International Conference on Intelligent Networks and Intelligent Systems (ICINIS), pages 370-373.

14) Kole, D.K. and Halder, A. (2010). An Efficient Dynamic Image Segmentation Algorithm using a Hybrid Technique based on PSO and Genetic Algorithm.International Conference on Advances in Computer Engineering ( $A C E$ ), pages 252-255.

15) Kulkarni, R. V., \& Venayagamoorthy, G. K. . (2010). Bio-inspired algorithms for autonomous deployment and localization of sensor nodes. IEEE Transactions, pages 663675.

16) Leung, Y., Zhang, J. and Xu, Z. (2000). Clustering by Space-Space Filtering. IEEE 
Transactions on Pattern Analysis and MAchine Intelligence, Vol 22, No. 12, pages 1396 1410.

17) Liu, A. and Wang, Y. Zhao, (2011). An Efficient Image Segmentation Method Based

on Fuzzy PSO and Markov Random Field Model. 7th International Conference on

Wireless Communications, Networking and Mobile Computing (WiCOM), pages 1-4.

18) Otsu, N. (1985). A Threshold Selection method from gray-level Histograms. IEEE

Transactions on Systems, Man and

Cybernetics, Vol. 9, No. 1, pages 62-66.

19) Puranik, P., Bajaj, P., Abraham, A., Palsodkar, P.and Deshmukh, A. (2009). Human Perception based Color Image Segmentation using Comprehensive Learning Particle Swarm Optimization. 2nd International Conference on Emerging Trends Engineering and Technology (ICETET), pages 630-635

20) Shen, S., Sandham, W., Granat, M. and Sterr, A. (2005). MRI Fuzzy

Segmentation of Brain Tissue using Neighbourhood Attraction with NeuralNetwork Optimization. IEEE Transactions on Information Technology in Biomedicine, Vol. 9, Issue 3, pages459. 467.

21) Singh, K. K. \& Singh, A. (2010, September). A Study of Image

Segmentation Algorithms for Different Types of Images. IJCSI International Journal of Computer Science Issues, Vol. 7, Issue 5, Pages 1-4.

22) Sonka, M., Hlavac, V. and Boyle, R. (1999). Image Processing Analysis and
Machine Vision. ITP, second edition

23) Tou, J. T. and Gonzalez, R. C. (1974). Pattern Recognitin Principles. Addison-

Wesley.

24) Turi, R. H. (2001). Clustering based Color Image Segmentation. Phd Thesis,

Monash University, Australia.

25) Wei, Z. and Zhu, Z. Y. (2010). Image Segmentation Algorithm based on Wavelet

Mutation Inertia Adaptive PSO. 29th Chinese Control Conference, pages 26902693.

26) Ying Ho, S. and Zheng Lee, K. (2003). Design and Analysis of an Efficient

Evolutionary Image Segmentation Algorithm. Journal if VISI Signal Processing, Vol 35, pages 29 - 42.

27) Yi, F., Chongxun, Z., Chen, P. and Li, L. (2005). White Blood Cell Image Segmentation using On-Line Trained Neural Network.27th Annual International Conference of the Engineering in Medicine and Biology (IEEE-EMBS), pages 6476-6479.

28) Zhang, Y., Huang, D., Ji, M. and Xie, F. (2011). Image Segmentation using PSO and PCM with Mahalanobis Distance. Expert Systems with Applications, Elsevier, Vol. 38, Issue 7, pages 90369040. 